\title{
If it is in the marrow, is it also in the blood? An analysis of 1,000 paired samples from patients with B-cell non-Hodgkin lymphoma
}

\author{
Patrizia Mancuso ${ }^{1}$, Angelica Calleri ${ }^{1}$, Pierluigi Antoniotti ${ }^{1}$, Jessica Quarna', Giancarlo Pruneri ${ }^{2}$, Francesco Bertolini ${ }^{1 *}$
}

\begin{abstract}
Background: Staging of B-cell non Hodgkin's lymphoma (NHL) routinely involves bone marrow (BM) examination by trephine biopsy (BM-TB). The evidence of disease in the BM-TB results in a clinical stage IV classification affecting therapeutic strategies for NHL patients. BM immunophenotyping by flow cytometry (FC) is also used, although its clinical value is still under debate.

Methods: Using FC we analyzed 1,000 paired BM aspirates and peripheral blood (PB) samples from $591 \mathrm{NHL}$ patients to investigate the concordance between BM and PB. B-lymphocytes were defined monoclonal when a ratio of $0.3<\kappa / \mathrm{I}>3$ was observed. Aberrant immunophenotypes present in the B-cell subpopulation were also investigated. BM-TB was also performed in $84.1 \%$ of samples (841/1000), and concordance between BM-TB and BM-FC was evaluated. Concordance was defined as the presence of a positive (in terms of disease detection) or negative result in both BM-FC and PB-FC or BM-TB and BM-FC.

Results: Using FC, the overall concordance between BM and PB was 95\%. Among the discordant cases (ie presence of neoplastic B-lymphocyte in the BM but under the sensibility of the technique in the PB) the most frequent diagnosis was Waldenstrom's macroglobulinemia (WM, accounting for $20.8 \%$ of all discordant cases). The expression of CXCR4, a receptor involved in B-cell trafficking and homing, was found to be down regulated in WM compared to other NHL types, thus suggesting a possible role of CXCR4 in WM cell homing in the BM. WM excluded, FC investigation of BM and PB in NHL patients gives overlapping information.

BM involvement was observed by FC in $38 \%$ of samples, and concordance between BM-FC and BM-TB was $85 \%$.
\end{abstract}

Conclusions: The finding that FC data from BM and PB samples overlap in NHL might have major implications for the design of future clinical studies and for patients' follow-up.

\section{Background}

Bone marrow (BM) examination by trephine biopsy (BM-TB) is routinely performed during staging and follow-up of patients affected by B-cell non-Hodgkin lymphoma (NHL). BM disease results in a stage IV classification, and may affect therapeutic strategies [1]. Flow cytometry BM immunophenotyping (BM-FC) is used in adjunct to BM-TB, even though its clinical value is still under investigation [2]. In the present study, in addition to the $\mathrm{BM}$ aspirate, the peripheral blood (PB)

\footnotetext{
* Correspondence: francesco.bertolini@ieo.it

'Laboratory of Hematology-Oncology, European Institute of Oncology, via Ripamonti 435, 20141 Milan, Italy

Full list of author information is available at the end of the article
}

was analyzed to investigate if malignant cells were restricted to BM or circulating in the blood.

Chemokine receptors are expressed by a variety of cells, including lymphoid cells, and mediate cell trafficking and homing. These receptors may also be involved in the migration and dissemination of NHL cells [3]. The stromal derived factor -1 (SDF-1, CXCL12) chemokine plays a crucial role in the retention of a variety of cells into BM niches through its receptor CXCR4 $[3,4]$. We investigated CXCR4 in different NHL subtypes to assess whether its expression correlates with differences in the frequency of NHL cells in the PB versus the BM. 


\section{Methods}

We retrospectively analyzed 1,000 paired $\mathrm{BM}$ aspirates and PB samples from 591 NHL patients (i.e. 1000 BM samples along with $1000 \mathrm{~PB}$ samples from the same day) consecutively collected in our Institute from 2000 to 2007 . BM-TB was also performed in $84.1 \%$ of paired samples $(841 / 1000)$, and, as BM-TB is considered the "gold-standard" for NHL staging and follow up, we also evaluated concordance between BM-TB and BM-FC.

Among the 1000 consecutive paired samples, 31\% were collected at the time of first diagnosis (616/2000), and $69 \%$ after therapy. For all patients, the diagnosis of NHL was obtained by morphology, phenotype and molecular analysis of nodal or extra-nodal sites and established according to the World Health Organization recommendations [5]. Hairy Cell Leukaemia, T-cell NHL, Hodgkin disease and multiple myeloma patients were not included in this study. The different subtypes of B-cell NHL are described in Table 1.

Four- (or, after 2005, six-) colour multiparametric FC was performed (Figure 1). Monoclonal antibodies including anti-CD45, -CD19, -CD20, surface IgM, $-\mathrm{CD} 10,-\mathrm{CD} 5,-\mathrm{CD} 43,-\mathrm{CD} 23$, anti- $\kappa$ and anti $-\lambda$ Ig light chain were used to analyze the B-lymphocyte immunophenotype. When light chain restriction was observed, anti-CD38, FMC-7, CD79b, CD22, CD103, CD11c, CD25 expression were also investigated on B-cells to better characterize B-cell phenotype [6] and to investigate the concordance between the diagnosis reported and the phenotype of the pathological B-lymphocytes observed. To detect light chain restriction, we used anti- $\kappa$ FITC/anti $-\lambda$ Pe/CD 45 PerCP/CD19 APC or anti- $\kappa$ FITC/anti $-\lambda$ Pe/CD45 PerCP/CD10 Pe-Cy-7/CD5 APC/ CD19 APC-Cy-7. As CD19-APC-Cy7 shows a very low signal-to-noise ratio in some cases like FL/DLBCL with low level CD19, the combination CD19 Pe/CD45 PerCP was used to compare the percentage of CD19 positive cells obtained with both markers. B-lymphocytes were defined monoclonal when a ratio of $0.3<\kappa / l>3$ was observed [7] or, for some lymphocytic lymphoma/ chronic lymphocytic leukaemia (CLL) patients, when surface membrane light chains were absent. At least $100 \mathrm{CD} 19+$ events showing the expected immunophenotype were required to define a $\mathrm{FC}$ test as positive [8]. In addition to this panel, anti-CD3,-CD4,-CD8 and $-\mathrm{CD} 16+56$ were routinely analyzed to gain information regarding the distribution of lymphocyte sub-populations. Controls included substitution of the relevant monoclonal antibody by a murine Ig of the same isotype. Monoclonal antibodies were purchased from BD Biosciences (Mountain View, CA), and used at the recommended concentration.

We also evaluated CXCR4 expression on B-cells in 54 paired BM-aspirates and PB (10 WM, 14 DLBC, $18 \mathrm{FL}$ and $12 \mathrm{CLL}$ ).

For CXCR4 detection, $10 \mu \mathrm{l}$ of PE-conjugated anti CXCR4 (BD, clone 12g5) was used in combination with anti CD45 Per-CP and CD19 Pe-Cy7. An isotypematched negative control was used. Mean fluorescence intensity (MFI) ratios were calculated by dividing the MFI for CXCR4 by the MFI of the respective isotypematched negative control, both in the BM aspirates and in PB. For sample preparation, a stain - lyse and wash standard procedure was used. Red cells were lysed by a $\mathrm{NH}_{4} \mathrm{Cl}$ solution. Measurements were performed on a FACS-Calibur or FACS-Canto (BD Biosciences), equipped with a second laser. Acquisition was stopped after collection of more than 200.000 total events or when at least 100 CD19 positive events were collected. Sensitivity of the procedure was established by serial dilution experiments as $1 / 10,000$.

BM-TB were fixed in $10 \%$ buffered formalin, decalcified with EDTA, embedded in paraffin and stained with haematoxylin-eosin. Immunohistochemistry with anti CD3-, CD5, CD10, CD20, CD23, CD34, CD43, CD79a, bcl-2, bcl-6, cyclin D1, Ki-67 was performed to distinguish between reactive and neoplastic lymphoid infiltration and to differentiate the different subtypes.

Table 1 Patient's Characteristics

\begin{tabular}{lc}
\hline Diagnosis & Number of Patients \\
\hline $\begin{array}{l}\text { Follicular Lymphoma } \\
\text { (FL) }\end{array}$ & 205 \\
\hline $\begin{array}{l}\text { Diffuse Large B cell Lymphoma } \\
\text { (DLBC-L) }\end{array}$ & 142 \\
\hline $\begin{array}{l}\text { Mantle Cell Lymphoma } \\
\text { (MCL) }\end{array}$ & 47 \\
\hline $\begin{array}{l}\text { Marginal Zone Lymphoma - Mucose Associated Lymphoma Tissue } \\
\text { (MZL-MALT) }\end{array}$ & 74 \\
\hline Lymphocytic lymphoma/chronic lymphocytic leukemia (CLL) & 83 \\
\hline $\begin{array}{l}\text { Waldenstrom's macroglobulinemia (WM) } \\
\text { TOTAL }\end{array}$ & 40 \\
\hline
\end{tabular}



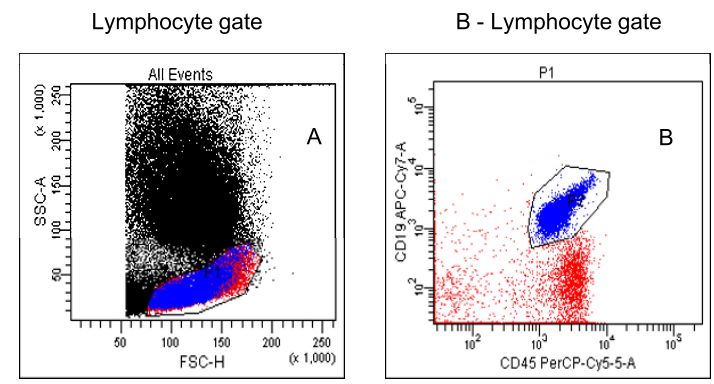

B - Lymphocyte phenotype

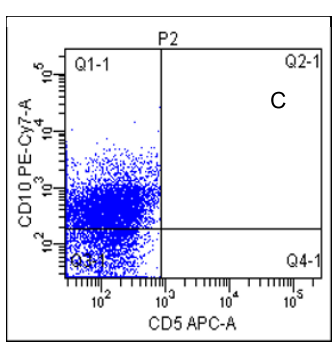

B - Lymphocyte chain restriction

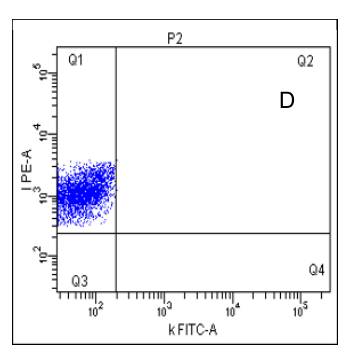

CXCR4 expression on B-lymphocytes

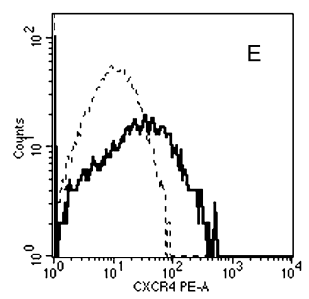

Follicular LNH

--- Waldenstrom's macroglobulinema

Figure 1 A-D: Representative FC dot plots showing the sequential multiparametric detection of clonal restriction in B-Lymphocytes from a patient with follicular NHL. Panel A shows the gate made on lymphocytes; Panel B shows CD19 and CD45 expression in the lymphocyte gate; Panel $\mathrm{C}$ shows the expression of CD5 and CD10; Panel D shows lambda chain clonal restriction; Panel E shows representative CXCR4 expression in patients with follicular LNH and WM.

Concordance was defined as the presence of a positive (in terms of disease detection) or negative result in both BM-FC and PB-FC or BM-TB and BM-FC. Statistical comparisons were performed using the t-test, analysis of variance (ANOVA) and linear regression when data were normally distributed and the non-parametric analyses of Spearman and Mann-Whitney when data were not normally distributed. Values of $\mathrm{p}$ lower than 0.05 were considered as significant.

\section{Results}

\section{Concordance between BM-FC and PB-FC}

As shown in Table 2, the most frequent diagnoses were Follicular NHL (FL, 35\%) and Diffuse Large B-Cell NHL (DLBC, 24\%). The overall FC concordance between BM and $\mathrm{PB}$ was $95.2 \%$ (Table 2) in all NHL subtypes, and the sensitivity of BM-FC was higher than that of PB-FC. In all discordant cases (4.8\%), monoclonal B lymphocytes were present in the BM, whereas in $\mathrm{PB}$ the monoclonal cell population was absent or under the sensitivity of the procedure. This was observed at diagnosis and after CT ( $\mathrm{p}<$ 0.001 in both cases). In discordant cases, BM monoclonal
B-cells ranged from $0.06 \%$ to $18 \%$ of all leucocytes. Among the 96 discordant cases, the most frequent diagnosis was Waldenstrom's macroglobulinemia (WM) (20.8\%). Sixty-two percent of discordant samples were collected after chemotherapy, and in discordant samples the most frequent treatment (38.5\% of all discordant cases) was Rituximab alone or in association with chemotherapy.

\section{Concordance between BM-TB and BM-FC}

Results of BM-TB are reported in Table 3. The concordance between BM-FC and BM-TB was $84 \%(83 \%$ at diagnosis and $85 \%$ after treatment). This result is in line with previous studies dealing with smaller series of patients that showed a 79-90\% concordance [9].

Among discordant cases (16\%, 135 samples, Figure 2), $75 \%$ of the samples (101 samples) were BM-FCpos/BMTBneg and 25\% (34 samples) were BM-FCneg/BMTBpos. Among BM-FCpos samples, 30\% (30 samples) were evaluated at first diagnosis (vs 36\%, 12 samples, of $\mathrm{BM}-\mathrm{TPpos}$ cases at first diagnosis, $\mathrm{p}=0.79$ ) and $70 \%$ (71 samples) were evaluated after therapy (vs 64\%, 22 samples, of BM-TBpos cases after therapy, $\mathrm{p}=0.89$ ). 
Table 2 Concordance between BM-FC and PB-FC

\begin{tabular}{|c|c|c|c|c|c|c|c|c|c|c|c|c|}
\hline & & \multicolumn{2}{|c|}{ Pts } & \multicolumn{2}{|c|}{ Samples } & \multicolumn{2}{|c|}{$\mathrm{BM}+\mathrm{PB}+$} & \multicolumn{2}{|c|}{ BM+PB- } & \multirow{2}{*}{$\begin{array}{c}\text { BM-PB+ } \\
\mathrm{N}^{\circ}\end{array}$} & \multicolumn{2}{|c|}{ BM-PB- } \\
\hline & & $\mathbf{N}^{\circ}$ & $\%$ & $\mathbf{N}^{\circ}$ & $\%$ & $\mathrm{~N}^{\circ}$ & $\%$ & $\mathbf{N}^{\circ}$ & $\%$ & & $\mathbf{N}^{\circ}$ & $\%$ \\
\hline \multirow[t]{3}{*}{$\mathrm{FL}$} & diag & & & 250 & $13 \%$ & 78 & $31,2 \%$ & 12 & $4,8 \%$ & 0 & 160 & $64,0 \%$ \\
\hline & after $\mathrm{CT}$ & & & 454 & $23 \%$ & 68 & $15,0 \%$ & 14 & $3,1 \%$ & 0 & 372 & $81,9 \%$ \\
\hline & both & 205 & $35 \%$ & 704 & $35 \%$ & 146 & $20,7 \%$ & 26 & $3,7 \%$ & 0 & 532 & $75,6 \%$ \\
\hline \multirow[t]{3}{*}{ DLBC } & diag & & & 146 & $7 \%$ & 22 & $15,1 \%$ & 10 & $6,8 \%$ & 0 & 114 & $78,1 \%$ \\
\hline & after $\mathrm{CT}$ & & & 218 & $11 \%$ & 56 & $25,7 \%$ & 4 & $1,8 \%$ & 0 & 158 & $72,5 \%$ \\
\hline & both & 142 & $24 \%$ & 364 & $18 \%$ & 78 & $21,4 \%$ & 14 & $3,8 \%$ & 0 & 272 & $74,7 \%$ \\
\hline \multirow[t]{3}{*}{$\mathrm{MCL}$} & diag & & & 24 & $1 \%$ & 21 & $87,5 \%$ & 1 & $4,2 \%$ & 0 & 2 & $8,3 \%$ \\
\hline & after $C T$ & & & 198 & $10 \%$ & 69 & $34,2 \%$ & 1 & $0,5 \%$ & 0 & 128 & $63,4 \%$ \\
\hline & both & 47 & $8 \%$ & 222 & $11 \%$ & 90 & $39,8 \%$ & 2 & $0,9 \%$ & 0 & 130 & $57,5 \%$ \\
\hline \multirow[t]{3}{*}{ MZL-MALT } & diag & & & 74 & $4 \%$ & 26 & $35,1 \%$ & 3 & $4,1 \%$ & 0 & 45 & $60,8 \%$ \\
\hline & after $\mathrm{CT}$ & & & 154 & $8 \%$ & 56 & $37,3 \%$ & 2 & $1,3 \%$ & 0 & 96 & $64,0 \%$ \\
\hline & both & 74 & $13 \%$ & 228 & $11 \%$ & 82 & $36,6 \%$ & 5 & $2,2 \%$ & 0 & 141 & $62,9 \%$ \\
\hline \multirow[t]{3}{*}{$C L L$} & diag & & & 78 & $4 \%$ & 74 & $94,9 \%$ & 0 & $0,0 \%$ & 0 & 4 & $5,1 \%$ \\
\hline & after $\mathrm{CT}$ & & & 236 & $12 \%$ & 152 & $64,4 \%$ & 14 & $5,9 \%$ & 0 & 70 & $29,7 \%$ \\
\hline & both & 83 & $14 \%$ & 314 & $16 \%$ & 226 & $72,0 \%$ & 14 & $4,5 \%$ & 0 & 74 & $23,6 \%$ \\
\hline \multirow[t]{3}{*}{ WM } & diag & & & 44 & $2 \%$ & 23 & $52,3 \%$ & 10 & $22,7 \%$ & 0 & 11 & $25,0 \%$ \\
\hline & after $\mathrm{CT}$ & & & 124 & $6 \%$ & 25 & $20,2 \%$ & 25 & $20,2 \%$ & 0 & 74 & $59,7 \%$ \\
\hline & both & 40 & $7 \%$ & 168 & $8 \%$ & 48 & $28,6 \%$ & 35 & $20,8 \%$ & 0 & 85 & $50,6 \%$ \\
\hline TOTAL & & 591 & $100 \%$ & 2000 & $100 \%$ & 670 & $33,5 \%$ & 96 & $4,8 \%$ & 0 & 1234 & $61,7 \%$ \\
\hline
\end{tabular}

Table 3 BM-TB results

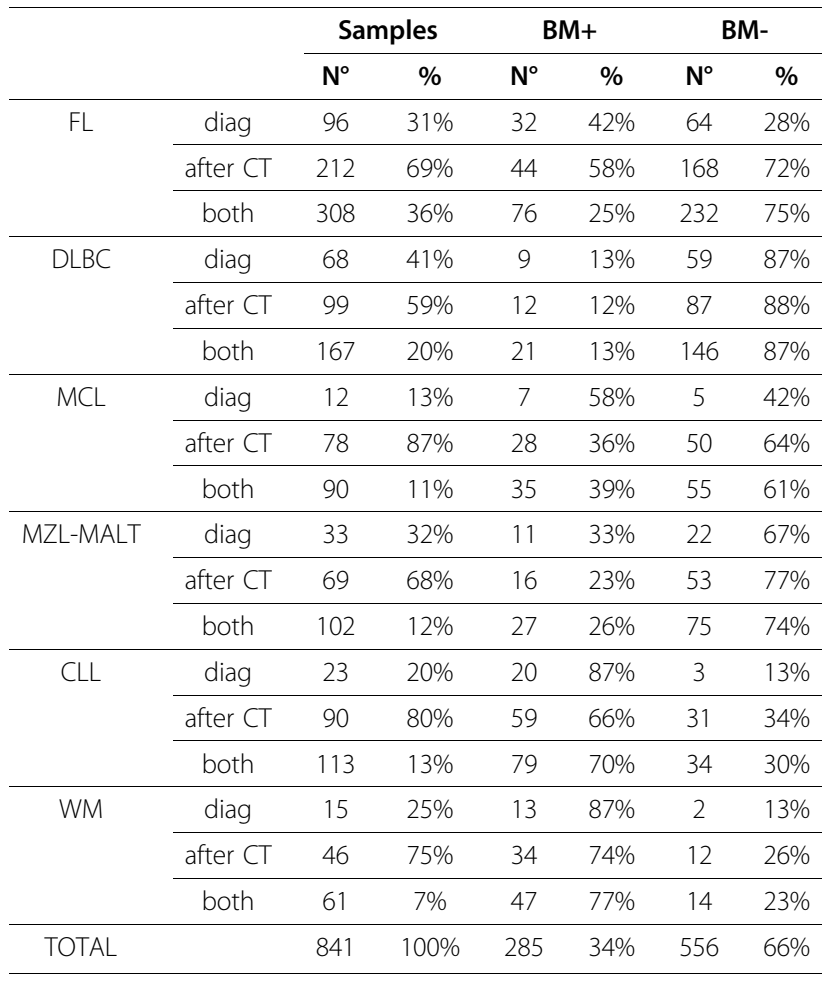

In BM-FCpos/BM-TBneg samples, the percentage of monoclonal $\mathrm{B}$ cells detected by FC was lower than $1 \%$ of all leukocytes, while in BM-FCneg/BM-TBpos samples, the most frequent finding was the presence of a nodular infiltrate. Differences in the frequency of positivity in the $\mathrm{BM}$ investigated by $\mathrm{FC}$ or $\mathrm{TB}$ were generally not significant, with the exceptions of MZL-MALT after therapy $(38.6 \%$ by $\mathrm{FC}$ vs $23 \%$ by $\mathrm{TB}, \mathrm{p}=0.036)$, and WM after therapy $(40.4 \%$ by FC vs $74 \%$ by $\mathrm{TB}, \mathrm{p}<$ $0.001)$. The difference observed in MCL at diagnosis was of borderline significance $(91.7 \%$ by FC vs $58 \%$ by $\mathrm{TB}, \mathrm{p}=0.053)$. At diagnosis, BM-TBneg/BM-FCpos cases were $0,8,33,6,8$, and $0 \%$ in FL, DLBC, MCL, MZL-MALT, CLL and WM, respectively. After CT, BM-TBneg/BM-FCpos cases were $0,14,0,15,4$, and $0 \%$ in FL, DLBC, MCL, MZL-MALT, CLL and WM, respectively.

\section{CXCR4 expression in different NHL subtypes}

In WM, we observed a significant CXCR4 downregulation in comparison to other NHL types (Figure 1). Median MFI ratio was 6 in WM vs 15,10 and 34 in DLBC-L, FL and CLL, respectively ( $\mathrm{p}<0.01$ ).

CXR4 expression in B lymphocytes in the BM and in the PB was not significantly different (data not shown). 


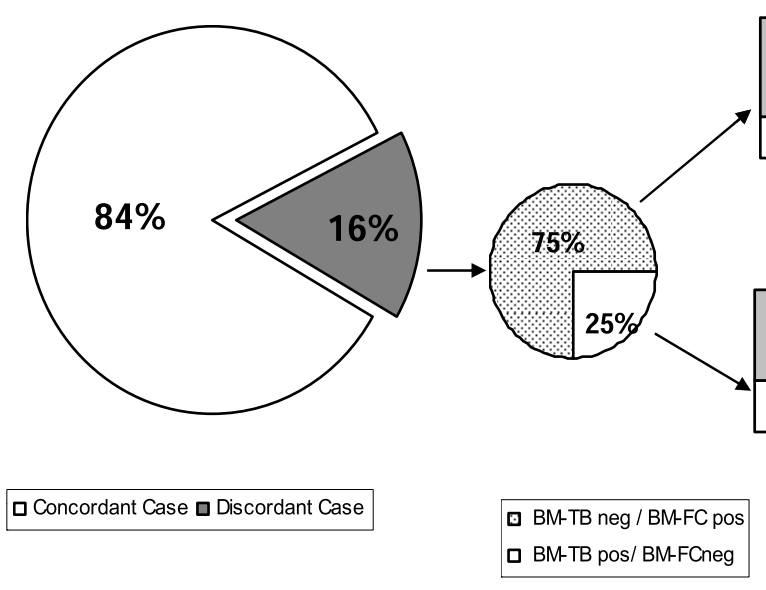

Figure 2 Analysis of discordance between BM-FC and BM-TB.

The crucial role of CXCR4 in WM cell homing in the marrow has been recently described [10]. Our current results suggest that CXCR4 expression is variable among different NHL subtypes, and lead to speculate a CXCR4 involvement in NHL blood spreading, possibly explaining the discordant results of $\mathrm{BM}$ and $\mathrm{PB}$ analyses in WM versus other NHL types. In fact, a lower CXCR4 MFI ratio was found in diseases where the presence of circulating neoplastic B cells is less frequent (WM and DLBC), and a higher ratio was found in NHL types associated with the presence of circulating neoplastic B cells in a larger frequency of cases (FL and CLL). Further studies are now needed to validate this hypothesis.

\section{Discussion}

$\mathrm{BM}$ examination by BM-TB is an integral part of the clinical staging and follow up of NHL patients. Along with $\mathrm{BM}-\mathrm{TB}, \mathrm{BM}-\mathrm{FC}$ is used as an ancillary investigation, even though its use is still debated. The correlation between these two techniques was found to be very high, and our results are in line with previous studies dealing with smaller series of patients (85\% vs $80 \%-90 \%$, see ref. [9]).

However, as BM aspiration might be a painful procedure for patients, we retrospectively analyzed 1,000 paired BM aspirates and PB samples from NHL patients to assess if $\mathrm{PB}$ analysis could be an alternative reliable procedure for staging and follow up of these patients. The overall FC concordance between BM and PB was $95.2 \%$. Among the discordant cases, $62 \%$ of samples were collected after chemotherapy and the most frequent treatment was Rituximab alone or in association with chemotherapy. This result suggests that during treatment with Rituximab alone or in association with chemotherapy, neoplastic B cells may be depleted in the $\mathrm{PB}$ but still present in the BM. Therefore, a negative $\mathrm{PB}$ sample obtained during treatment with Rituximab should be considered with caution [8].

Among discordant cases, the most frequent diagnosis was WM (20.8\%). Among the different biological reasons that might explain this discrepancy, we focused on CXCR4. The crucial role of CXCR4 in WM cell homing in the BM has been recently described [10] and our results, even though obtained in a small number of patients and samples, confirm this hypothesis. In fact, CXCR4 expression on B lymphocytes was found to be down-regulated when compared to other NHL types $(p<0.001)$.

Albeit the investigation of NHL patients' clinical outcome was beyond the scope of the present study, encompassing a large variety of NHL types and treatment plans, we tried to investigate the clinical outcome of the discordant cases. However, the clinical follow-up of the discordant cases did not offer any meaningful and significant information, because of the very large variety of different treatment procedures in the patients.

To the best of our knowledge, only two previous papers have reported about the role of FC in clinical NHL outcome. Perea et al [9] reported discordance between BM-FC and BM-TB (BM-FCpos/BM-TBneg) in 9\% (36) of low-grade NHL patients, in most cases (66\%) affected by FL. In this study, discordance had no apparent influence on the clinical outcome when compared with BM-negative patients (median follow-up 14 months). In the second study, Gronich et al [11] investigated 70 low-grade NHL patients and reported that the outcome in patients who had BM involvement defined by FC alone or by morphology was similar.

\section{Conclusions}

Our data indicate that - WM excluded - FC investigation of $\mathrm{BM}$ and $\mathrm{PB}$ in NHL patients gives overlapping 
information. This finding might have major implications for the design of future NHL clinical studies and suggest that the FC investigation of $\mathrm{PB}$ has potential as a possible alternative to BM-TB for the follow-up of NHL patients.

\section{Acknowledgements}

Supported in part by AIRC (Associazione Italiana per la Ricerca sul Cancro), and ISS (Istituto Superiore di Sanità).

\section{Author details}

'Laboratory of Hematology-Oncology, European Institute of Oncology, via Ripamonti 435, 20141 Milan, Italy. ${ }^{2}$ Division of Pathology-Laboratory Medicine, European Institute of Oncology, via Ripamonti 435, 20141 Milan, Italy.

\section{Authors' contributions}

PM, GP, and FB: designed the research, analyzed the data, and wrote the paper.

AC, PA, JQ performed flow cytometric analysis, and analyzed the data. All authors read and approved the final manuscript.

\section{Competing interests}

The authors declare that they have no competing interests.

Received: 17 May 2010 Accepted: 24 November 2010

Published: 24 November 2010

\section{References}

1. Yan Y, Chan WC, Weisenburger DD, Anderson JR, Bast MA, Vose JM, Bierman PJ, Armitage JO: Clinical and prognostic significance of bone marrow involvement in patients with diffuse aggressive B-cell lymphoma. Journal of Clinical Oncology 1995, 13:1336-1342.

2. Cheson BD, Pfistner B, Juweid ME, Gascoyne RD, Specht L, Horning SJ, Coiffier B, Fisher Rl, Hagenbeek A, Zucca E, Rosen ST, Stroobants S, Lister TA, Hoppe RT, Dreyling M, Tobinai K, Vose JM, Connors JM, Federico M, Diehl V: Revised response criteria for malignant lymphoma. Journal of Clinical Oncology 2007, 25:579-586.

3. Laurence AD: Location, movement and survival: the role of chemokines in haematopoiesis and malignancy. British Journal of Haematology 2006, 132:255-267.

4. Bertolini F, Dell'Agnola C, Mancuso P, Rabascio C, Burlini A, Monestiroli S, Gobbi A, Pruneri G, Martinelli G: CXCR4-neutralization, a novel therapeutic approach for non-Hodgkin lymphoma. Cancer Research 2002, 62:3106-3112.

5. Harris NH, Jaffe ES, Diebold J, Flandrin G, Muller-Hermelink HK, Vardiman J, Lister TA, Bloomfileld CD: World Health Organization Classification of neoplastic diseases of the hematopoietic and lymphoid tissues: Report of the clinical advisory committee meeting - Airlie House, Virginia, November 1997. Journal of Clinical Oncology 1999, 17:3835-3849.

6. Sánchez ML, Almeida J, Vidriales B, López-Berges MC, García-Marcos MA, Moro MJ, Corrales A, Calmuntia MJ, San Miguel JF, Orfao A: Incidence of phenotypic aberrations in a series of 467 patients with $B$ chronic lymphoproliferative disorders: basis for the design of specific four-color stainings to be used for minimal residual disease investigation. Leukemia 2002, 16:1460-1469.

7. Hanson CA, Kurtin PJ, Jerry A, Katzmann JA, Hoyer JD, Li CY, Hodnefield JM, Meyers CH, Habermann TM, Witzig TE: Immunophenotypic Analysis of Peripheral Blood and Bone Marrow in the Staging of B-Cell Malignant Lymphoma. Blood 1999, 94:3889-3896.

8. Rawstron AC, Villamor N, Ritgen M, Böttcher S, Ghia P, Zehnder $J$, Lozanski G, Colomer D, Moreno C, Geuna M, Evans PA, Natkunam Y, Coltre SE, Avery ED, Rassenti LZ, Kipps TJ, Caligaris-Cappio F, Kneba M, Byrd JC, Hallek MJ, Montserrat E, Hillmen P: International standardized approach for flow cytometric residual disease monitoring in chronic lymphocytic leukaemia. Leukemia 2007, 21:956-964.

9. Perea G, Altes A, Bellido M, Aventin A, Bordes R, Ayats R, Remacha AF, Espinosa I, Briones J, Sierra J, Nomdedeu JF: Clinical utility of bone marrow flow cytometry in B-cell non-Hodgkin lymphomas. Histopathology 2004, 45:268-274.

10. Ngo HT, Leleu X, Lee J, Jia X, Melhem M, Runnels J, Moreau AS, Burwick N, Azab AK, Roccaro A, Azab F, Sacco A, Farag M, Sackstein R, Ghobrial IM: SDF-1/CXCR4 and VLA-4 interaction regulates homing in Waldenstrom macroglobulinemia. Blood 2008, 112:150-158.

11. Gronich N, Radnay J, Shapiro H, Manor Y, Lahav M, Lishner M: Clinical outcome of low-grade NHL patients with bone marrow involvement. European Journal of Clinical Investigation 2007, 37:05-309.

\section{Pre-publication history}

The pre-publication history for this paper can be accessed here: http://www.biomedcentral.com/1471-2407/10/644/prepub

doi:10.1186/1471-2407-10-644

Cite this article as: Mancuso et al: If it is in the marrow, is it also in the blood? An analysis of 1,000 paired samples from patients with B-cell non-Hodgkin lymphoma. BMC Cancer 2010 10:644.

\section{Submit your next manuscript to BioMed Central and take full advantage of:}

- Convenient online submission

- Thorough peer review

- No space constraints or color figure charges

- Immediate publication on acceptance

- Inclusion in PubMed, CAS, Scopus and Google Scholar

- Research which is freely available for redistribution 